\title{
openheart Comparative early outcomes of tricuspid Valve repair versus replacement for secondary tricuspid regurgitation
}

Mohamad Alkhouli, Chalak Berzingi, Amer Kowatli, Fahad Alqahtani, Vinay Badhwar

\begin{abstract}
- Additional material is published online only. To view please visit the journal online (http://dx.doi.org/10.1136/ openhrt-2018-000878).
\end{abstract}

To cite: Alkhouli M, Berzingi C, Kowatli A, et al. Comparative early outcomes of tricuspid Valve repair versus replacement for secondary tricuspid regurgitation. Open Heart 2018;5:e000878. doi:10.1136/ openhrt-2018-000878

Received 8 June 2018 Revised 18 July 2018 Accepted 14 August 2018
Check for updates

(C) Author(s) (or their employer(s)) 2018. Re-use permitted under CC BY-NC. No commercial re-use. See rights and permissions. Published by BMJ.

West Virginia University Heart and Vascular Institute, Morgantown, West Virginia, USA

Correspondence to Dr Mohamad Alkhouli; adnanalkhouli@gmail.com

\section{ABSTRACT}

Background Comparative outcome data on tricuspid valve repair (TVr) versus tricuspid valve replacement (TVR) for severe secondary tricuspid regurgitation (TR) are limited.

Methods We used a national inpatient sample to assess in-hospital morbidity and mortality, length of stay and cost in patients with severe secondary TR undergoing isolated TVr versus TVR.

Results A total of 1364 patients (national estimate $=6757$ ) underwent isolated tricuspid valve surgery during the study period, of whom 569 (41.7\%) had TVr and $795(58.3 \%)$ had TVR. There was no difference in the prevalence of major morbidities between the two groups, except for liver disease and hepatic cirrhosis, which were more common in the TVR group. Before propensity matching, in-hospital mortality was similar between patients who underwent isolated TVr and TVR $(8.1 \%$ vs $10.8 \%, p=0.093)$, but the incidence of postoperative morbidities differed: TVR was associated with higher rates of permanent pacemaker implantation and blood transfusion, while TVr was associated with more acute kidney injury. After rigorous propensity score matching, TVR was associated with significantly higher rates of inhospital death ( $12 \%$ vs $6.9 \%, p=0.009)$ and permanent pacemaker implantation $(33.7 \%$ vs $11.2 \%, \mathrm{p}<0.001)$. Postoperative morbidities and length of stay, however, were not different between the two groups. Nonetheless, cost of hospitalisation was $16 \%$ higher in the TVr group. Conclusions In patients undergoing isolated surgery for secondary TR, TVR is associated with higher in-hospital mortality and need for permanent pacemaker compared with TVr. Further studies are needed to understand the impact of the type of surgery on the short-term and longterm mortality in this complex undertreated population.

Secondary tricuspid regurgitation (TR) is the predominant cause of severe TR in North America. ${ }^{1}$ Despite the deleterious effect of severe TR on long-term outcomes, contemporary data on tricuspid valve (TV) surgery remain limited. $^{2}$ This is likely multifactorial due to modest annual volume of TV surgery, under-recognition of TV disease, delayed

\section{Key questions}

What is already known about this subject?

- Comparative outcomes data for tricuspid valve repair versus replacement inpatients with severe secondary tricuspid regurgitation are limited.

What does this study add?

- In patients with severe secondary tricuspid regurgitation, tricuspid valve replacement is associated with significantly higher rates of in-hospital death ( $12 \%$ vs $6.9 \%, p=0.009$ ) and permanent pacemaker implantation $(33.7 \%$ vs $11.2 \%, p<0.001)$.

How might this impact on clinical practice?

- In patients with severe secondary tricuspid regurgitation and suitable anatomy, tricuspid repair is associated with at least comparable outcomes compared with valve replacement.

- However, further comparative studies are needed to assess long-term outcomes of patients undergoing repair versus replacement.

clinical presentation, high prevalence of comorbidities in these patients, and lack of consensus on the approach and timing of TV surgery. ${ }^{3}$ Once the patient is referred for TV surgery for severe TR, the decision to repair or replace the valve is also complex and depends on the patient's age and comorbidities, the degree of right ventricular dilatation and dysfunction, the need for a concomitant procedure, and surgical expertise. Comparative outcome data on tricuspid valve repair (TVr) versus tricuspid valve replacement (TVR) for secondary TR are limited. A recent meta-analysis by Choi et at suggested more favourable outcomes with $\mathrm{TVr}$, but this was confounded with the inclusion of patients with primary TR and concomitant operations. The purpose of this study is to assess in-hospital morbidity and mortality, length of stay and cost in patients with severe secondary 
TR undergoing isolated TVr versus TVR using a contemporary national representative database.

\section{METHODS}

The Nationwide Inpatient Sample (NIS) ${ }^{3}$ was used to derive patient-relevant information between January 2003 and December 2014. The NIS is the largest, publicly available, all-payer administrative claims-based database and contains information about patient discharges from $\sim 1000$ non-federal hospitals in 45 states. It contains clinical and resource use information on 5-8 million discharges annually, with safeguards to protect the privacy of individual patients, physicians and hospitals. The NIS shares certain similarities with the Medicare database, including the same International Classification of Diseases, Ninth Revision, Clinical Modification (ICD-9-CM) coding system for procedures and diagnoses. Contrary to the Medicare database, the NIS includes all payers and patients across all ages. These data are stratified to represent $\sim 20 \%$ of US inpatient hospitalisations across different hospital and geographical regions (random sample). The national estimates (NE) represent a calculated estimate of the total $(100 \%)$ US hospitalised population. This is calculated using the Agency for Healthcare Research and Quality sampling and weighting method. Outcomes analysis was performed using the actual $20 \%$ sample available in the NIS, whereas the trend analysis was performed using the NE.

Patients aged 18 years and older who underwent TVR (ICD-9-CM procedure code 35.27 and 35.28) and TVr (ICD-9-CM code 35.14) during the study period were identified. To identify patients with secondary TR, those with congenital TV disease (ICD-9-CM codes 764.1, 746.2, 745.4 and 746.89) or those with infective endocarditis (ICD-9-CM code 571.2) were excluded. Patients who underwent redo TV surgery were also excluded (ICD-9-CM codes 35.20 and 35.21). To eliminate potential impact of concurrent cardiac surgery on outcomes, patients who underwent a concomitant valve surgery, coronary bypass grafting or surgical ablation for atrial fibrillation (maze procedure) were excluded. A flow chart of the study is provided in figure 1.

Patients who underwent isolated TV surgery for severe secondary TR were divided into two groups according to the surgical approach (group 1, isolated TVr; and group 2 , isolated TVR). We then performed a comparative analysis between the outcomes of isolated TVr and TVR in patients with severe secondary TR. To account for potential confounding factors and reduce the effect of selection bias, a propensity score matching model was developed using logistic regression to derive two matched groups for comparative outcomes analysis. Patients who underwent isolated TVr or isolated TVR were entered into a nearest neighbour 1:1 variable ratio, parallel, balanced propensity matching model using a calliper of 0.01 to attain two pairs of well-matched cohort for outcomes analysis. Propensity scores were derived from 41 hospital, clinical and demographic covariates, including the Elixhauser Comorbidity Index. The primary endpoint was in-hospital death. The secondary outcomes included postoperative morbidities, length of stay, hospital charges and discharge disposition.

Patient-relevant descriptive statistics are presented as frequencies with percentages for categorical variables and as means with SD for continuous variables. Baseline characteristics were compared between the groups using a Pearson's $\chi^{2}$ test for categorical variables and an independent-samples t-test for continuous variables. A Cochran-Armitage test was used to evaluate trends in isolated TVr and isolated TVR in patients with severe secondary TR. Matched categorical variables were presented as frequencies with percentages and compared using McNemar's test. Matched continuous variables were presented as means with SD and compared using a pair ed-samples t-test. All statistical analyses were performed using SPSS V.24 and R V.3.3.1.

\section{RESULTS}

A total of 1364 actual reported cases $(\mathrm{NE}=6757)$ underwent isolated TV surgery during the study period, of whom 569 (41.7\%) had TVr and 795 (58.3\%) had TVR (figure 2). Compared with patients who had TVr, those who underwent TVR were older $(56 \pm 17$ vs $54 \pm 18$, $\mathrm{p}=0.02)$, were women $(57.6 \%$ vs $51.1 \%, \mathrm{p}=0.018)$ and were Caucasians $(71.9 \%$ vs $64.6 \%, \mathrm{p}=0.018)$. There was no difference in the prevalence of major morbidities between the two groups (table 1), except for liver disease and hepatic cirrhosis, which were more common in the TVR group ( $11.1 \%$ vs $3.7 \%$ and $5.8 \%$ vs $1.4 \%$, respectively, $\mathrm{p}<0.001$ for all). Surgery was frequently performed during a non-elective admission in both groups but more commonly for $\mathrm{TVr}(45.8 \%$ vs $35 \%$, $\mathrm{p}<0.001)$.

Before propensity score matching, in-hospital mortality was similar between patients who underwent isolated TVr or TVR ( $8.1 \%$ vs $10.8 \%, p=0.093)$, but the incidence of postoperative morbidities differed: TVR was associated with a threefold higher rate of permanent pacemaker implantation $(34.1 \%$ vs $10.9 \%, \mathrm{p}<0.001)$ and a trend towards more blood transfusion $(37.6 \%$ vs $32.7 \%$, $\mathrm{p}=0.06$ ), while $\mathrm{TVr}$ was associated with more acute kidney injury ( $33.7 \%$ vs $27.8 \%, \mathrm{p}=0.018)$. Nonetheless, the need for renal replacement therapy following surgery was not different between the two groups ( $4.4 \%$ vs $5.5 \%$ following TVr vs TVR, $\mathrm{p}=0.34$ ). Patients who had TVr had longer hospitalisations $(23 \pm 26$ vs $19 \pm 24, \mathrm{p}=0.013)$, but both groups had comparable rates of non-home discharges (table 2). Temporal trends in in-hospital mortality following isolated TVR and TVr are shown in (online supplementary figure 1 ).

After rigorous propensity score matching, no significant differences in baseline characteristic were observed (table 1). In propensity-matched patients, TVR was associated with significantly higher rates of in-hospital death (12\% vs 6.9\%, p=0.009) and permanent pacemaker 


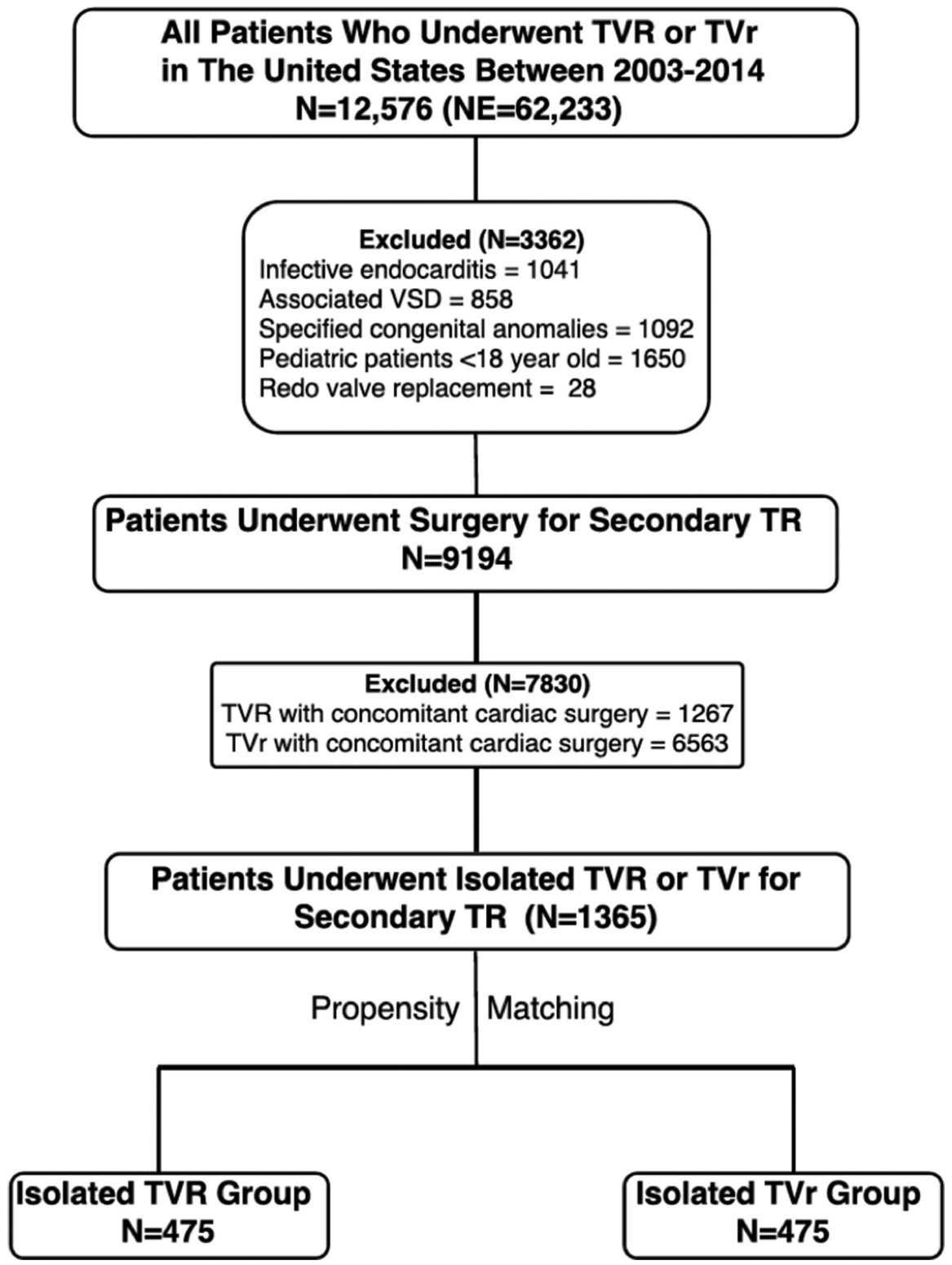

Figure 1 Study flow chart. NE, national estimate; TR, tricuspid regurgitation; TVr, tricuspid valve repair; TVR, tricuspid valve replacement.

implantation $(33.7 \%$ vs $11.2 \%, \mathrm{p}<0.001)$. Postoperative morbidities and length of stay, however, were not different between the two groups (table 2). Nonetheless, cost of hospitalisation was $16 \%$ higher in the TVr group.

\section{DISCUSSION}

The following are the main findings of the present investigation: (1) Isolated tricuspid surgery for secondary TR is infrequently performed in the USA. (2) Patients undergoing isolated TVR versus TVr have similar baseline characteristics, with the exception of chronic liver disease, which was more prevalent in the TVR group. (3) In propensity-matched cohorts of patients, isolated TVR is associated with $74 \%$ higher incidence of in-hospital mortality and $300 \%$ higher rate of permanent pacemaker implantation compared with isolated TVr. Although length of stay was comparable in both groups, cost of hospitalisation was $16 \%$ higher after TVr than after TVR.

It is estimated that 1.6 million patients in the USA suffer from moderate to severe TR. ${ }^{56}$ In the Framingham Study moderate or severe TR was present in $1.5 \%$ of men and $5.6 \%$ of women $>70$ years of age. ${ }^{7}$ The prevalence is higher in patients with concomitant valvular disease or cardiomyopathy. Moderate to severe TR is found in $30 \%-50 \%$ of patients with severe mitral regurgitation, $12 \%-25 \%$ of patients with severe aortic stenosis and $19 \%$ of patients with congestive heart failure. ${ }^{8-12}$ The percentage of patients with symptomatic TR is uncertain and remains difficult to estimate given the high prevalence of other potential sources of symptoms and comorbidities in these patients. ${ }^{13}$ Nonetheless, the current study confirms that only a small fraction of patients at 


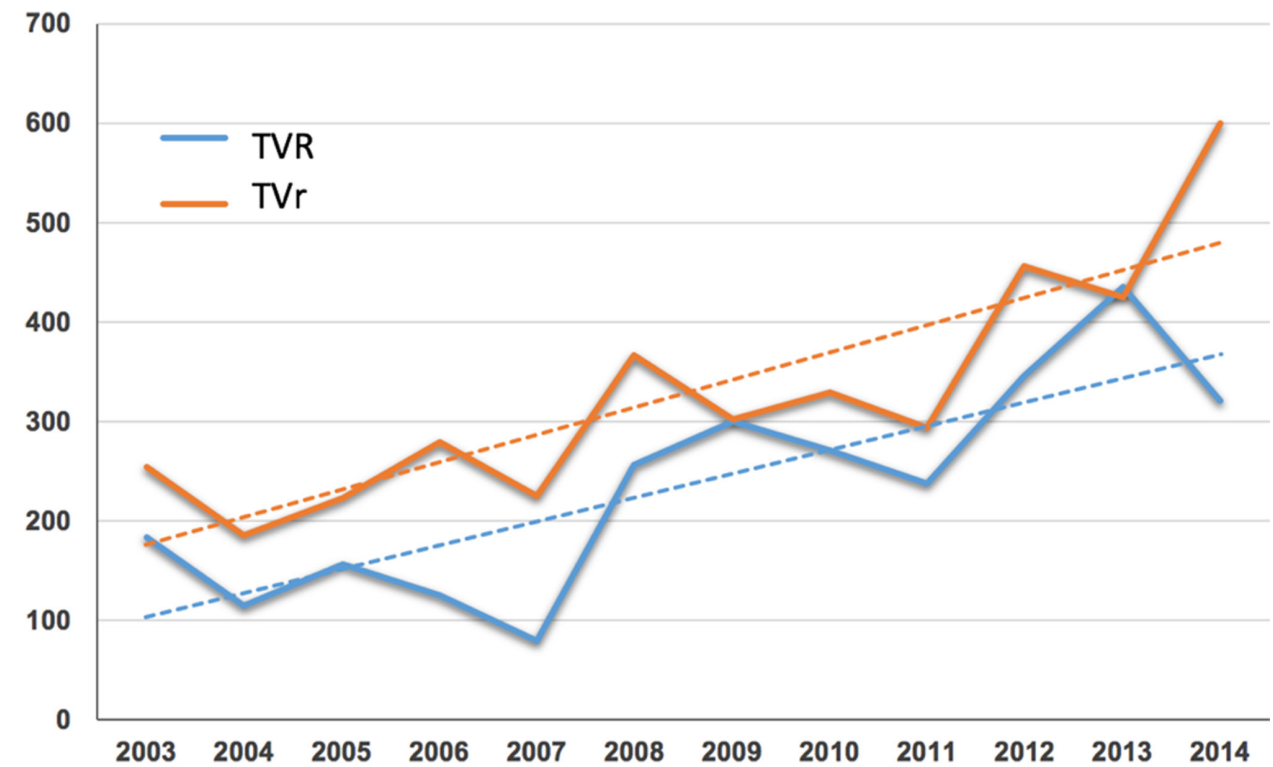

figure 2 Temporal trend of isolated tricuspid valve surgery for secondary tricuspid regurgitation in the USA between 2003 and 2014. TVr, tricuspid valve repair; TVR, tricuspid valve replacement. $x$ axis, year; y axis, number of cases (weighted national estimates).

risk undergo isolated TV surgery in the USA. Among these patients, however, a slight majority undergoes TVR $(58.3 \%)$ and the remainder undergo TVr.

Despite the relatively young age of patients in this cohort ( $55 \pm 17$ years), major comorbidities were common (hypertension $43 \%$, diabetes $18 \%$, atrial fibrillation $45 \%$, coagulopathy $28 \%$ and chronic renal disease $22 \%)$. Although both groups had comparable baseline risk profiles, the TVR group had higher prevalence of chronic liver disease. This is important and this could be related to a more advanced right ventricular disease and subsequent congestive hepatopathy, both are likely to contribute to worse short-term and long-term outcomes in the TVR group.

The decision of whether to repair or replace the TV is complex and depends on multiple factors: (1) timing of surgery, (2) anatomical factors and (3) clinical expertise. Unlike aortic or mitral valve disease, where the timing of operation may often be driven by symptoms, many patients with severe TR remain symptomatic for prolonged periods before being referred to surgical correction. At this point, patients have often developed severe tethering and marked right ventricular dysfunction that may inevitably impact surgical outcomes. Anatomical factors, such as the degree of annular dilatation and leaflet tethering, play a significant role in determining the surgical therapy. Although TVr is usually the preferred approach in order to minimise bypass time, TVR is frequently needed in cases of extreme annular dilatation or leaflet abnormality (such as in pacemaker-related TR) ${ }^{14} 15$ Patient choice, compliance and clinical expertise also influence the choice of TVr versus TVR. Bioprosthetic valve degeneration and mechanical valve thrombosis continue to generate much debate and may impact the decision to repair or replace in patients with compliance issues. ${ }^{16}$
Similarly, experience with repair techniques is key to minimise risk of recurrence. ${ }^{17}$ The lack of such experience may also affect decision making on the surgical approach. Hence, selection bias will remain a significant confounder in any retrospective investigations pertaining to TV surgery. Notably, a high percentage of patients had bioprosthetic valve implantation in this study, likely due to concerns about non-compliance with lifelong anticoagulation. Although we performed vigorous propensity score matching to account for differences in baseline risk profiles and hospital characteristics, the above-mentioned factors should be considered when interpreting the current study results.

In propensity-matched groups, TVR was associated with higher in-hospital mortality compared with TVr. Prior comparative studies on TVr versus TVR yielded conflicting data likely due to design issues and the inclusion of heterogeneous groups of patients with various TR aetiologies. ${ }^{18-27}$ A recent comprehensive meta-analysis pooled data from 17 studies and compared TVr versus TVR in patients with severe TR for the primary endpoint of all-cause mortality. ${ }^{4}$ This meta-analysis showed that TVR was associated with higher mortality compared with $\mathrm{TVr}$ (HR 1.59, 95\% CI 1.26 to 2.00) $\left(\mathrm{I}^{2}=62 \%\right)$. A subanalysis including only studies with predominantly patients with secondary TR revealed that TVR remained associated with higher odds of all-cause mortality (HR 1.39, 95\% CI 1.04 to 1.86 ). This is consistent with the current findings of a $74 \%$ higher risk of postoperative mortality following TVR versus TVr. The contribution of right ventricular dysfunction, duration of the TV disease, and surgical volume and techniques on this excess mortality in the TVR group cannot be ascertained due to the lack of data on disease duration or echocardiographic findings in the NIS and 
Table 1 Characteristics of unmatched and propensity-matched patients undergoing isolated tricuspid repair versus replacement, 2003-2014

\begin{tabular}{|c|c|c|c|c|c|c|}
\hline \multirow[b]{2}{*}{$\begin{array}{l}\text { Baseline } \\
\text { characteristics }\end{array}$} & \multicolumn{3}{|c|}{ Unmatched cohorts } & \multicolumn{3}{|c|}{ Matched cohorts } \\
\hline & $\begin{array}{l}\text { TVr } \\
\mathrm{n}=569\end{array}$ & $\begin{array}{l}\text { TVR } \\
\mathrm{n}=795\end{array}$ & P values & $\begin{array}{l}\text { TVr } \\
\mathrm{n}=475\end{array}$ & $\begin{array}{l}\text { TVR } \\
\mathrm{n}=475\end{array}$ & $P$ values \\
\hline Female, n (\%) & $291(51.1)$ & $458(57.6)$ & 0.018 & $259(54.5)$ & $259(54.5)$ & 0.99 \\
\hline Hispanic race & $35(7.4)$ & $49(7.6)$ & & $39(8.2)$ & $37(7.8)$ & \\
\hline \multicolumn{7}{|l|}{ Medical comorbidity } \\
\hline Hypertension & $241(42.4)$ & $346(44)$ & 0.56 & $209(44)$ & $205(43.2)$ & 0.838 \\
\hline Diabetes & $103(18.1)$ & $140(17.6)$ & 0.815 & 85 (17.9) & $84(17.7)$ & 0.99 \\
\hline Anaemia & $120(21.1)$ & $175(22)$ & 0.683 & $99(20.8)$ & $99(20.8)$ & 0.99 \\
\hline Coagulopathy & $155(27.3)$ & $219(27.9)$ & 0.816 & $129(27.2)$ & $131(27.6)$ & 0.943 \\
\hline $\begin{array}{l}\text { Conduction } \\
\text { abnormalities }\end{array}$ & $17(3)$ & $23(2.9)$ & 0.919 & $11(2.3)$ & $12(2.5)$ & 0.99 \\
\hline Vascular disease & $37(6.5)$ & $54(6.8)$ & 0.832 & $33(6.9)$ & $29(6.1)$ & 0.688 \\
\hline $\begin{array}{l}\text { Chronic renal } \\
\text { disease }\end{array}$ & $125(22)$ & $183(23)$ & 0.647 & $100(21.1)$ & $101(21.3)$ & 0.99 \\
\hline Haemodialysis & $17(3)$ & $36(4.5)$ & 0.147 & $17(3.6)$ & $18(3.8)$ & 0.99 \\
\hline Large hospital size & $494(87.1)$ & $650(82)$ & 0.033 & $406(85.5)$ & $400(84.2)$ & 0.342 \\
\hline Rural location & $6(1.1)$ & $9(1.1)$ & 0.894 & $6(1.3)$ & $6(1.3)$ & 0.99 \\
\hline $\begin{array}{l}\text { Non-elective } \\
\text { admission }\end{array}$ & $260(45.8)$ & $278(35)$ & $<0.001$ & $187(39.4)$ & $179(37.7)$ & 0.622 \\
\hline $\begin{array}{l}\text { Mechanical } \\
\text { prosthesis }\end{array}$ & $0(0)$ & $305(38.4)$ & $<0.001$ & $0(0)$ & $203(42.7)$ & $<0.001$ \\
\hline Primary payer, n (\%) & & & 0.019 & & & 0.595 \\
\hline $\begin{array}{l}\text { Medicare/ } \\
\text { Medicaid }\end{array}$ & $315(55.4)$ & $506(63.6)$ & & $275(57.9)$ & $271(57.1)$ & \\
\hline $\begin{array}{l}\text { Private, including } \\
\text { HMO }\end{array}$ & $214(37.6)$ & $240(30.2)$ & & $169(35.6)$ & $168(35.4)$ & \\
\hline $\begin{array}{l}\text { Self-pay/no } \\
\text { charge/other }\end{array}$ & $18(3.2)$ & $25(3.1)$ & & $15(3.2)$ & $16(3.4)$ & \\
\hline Median income, n (\%) & & & 0.565 & & & 0.437 \\
\hline $\begin{array}{l}1.0-25 \text { th } \\
\text { percentile }\end{array}$ & $159(28.7)$ & $200(25.9)$ & & $135(28.4)$ & $126(26.5)$ & \\
\hline $\begin{array}{l}2.26-50 \text { th } \\
\text { percentile }\end{array}$ & $128(23.1)$ & $196(25.4)$ & & $113(23.8)$ & $127(26.7)$ & \\
\hline
\end{tabular}


Table 1 Continued

\begin{tabular}{|c|c|c|c|c|c|c|}
\hline \multirow[b]{2}{*}{$\begin{array}{l}\text { Baseline } \\
\text { characteristics }\end{array}$} & \multicolumn{3}{|c|}{ Unmatched cohorts } & \multicolumn{3}{|c|}{ Matched cohorts } \\
\hline & $\begin{array}{l}\text { TVr } \\
n=569\end{array}$ & $\begin{array}{l}\text { TVR } \\
n=795\end{array}$ & P values & $\begin{array}{l}\text { TVr } \\
n=475\end{array}$ & $\begin{array}{l}\text { TVR } \\
n=475\end{array}$ & $\mathbf{P}$ values \\
\hline $\begin{array}{l}3.51-75 \text { th } \\
\text { percentile }\end{array}$ & $137(24.7)$ & $184(23.8)$ & & $111(23.4)$ & $105(22.1)$ & \\
\hline $\begin{array}{l}4.76-100 \text { th } \\
\text { percentile }\end{array}$ & $130(23.5)$ & $193(25)$ & & $116(24.4)$ & $117(24.6)$ & \\
\hline
\end{tabular}

$\mathrm{HMO}$, health maintenance organisation;TVR, tricuspid valve replacement;TVr, tricuspid valve repair.

the inability to perform a meaningful volume-outcomes analysis due to the very low volume of TV surgery in the USA overall. Some of these factors, however, have been shown to play an important role on the operative mortality following TV surgery. The role of routine surveillance and early intervention was demonstrated in a Korean registry of 106 patients with rheumatic valvular disease and prior left-sided valve operations.
These patients had worsening TR during follow-up and subsequently underwent TVR (50\%) and TVr (50\%), which were performed with a very low early operative mortality $(1.9 \%) .^{28}$ Similar low operative mortality of TVR and TVr has been demonstrated in other series. ${ }^{25} 29$ In the study by Sung et $a l,{ }^{29}$ the authors suggested their liberal use of modified ultrafiltration (95\% of patients) was essential in reducing postoperative mortality. The

Table 2 In-hospital outcomes of unmatched and propensity -matched patients undergoing isolated tricuspid repair versus replacement, 2003-2014

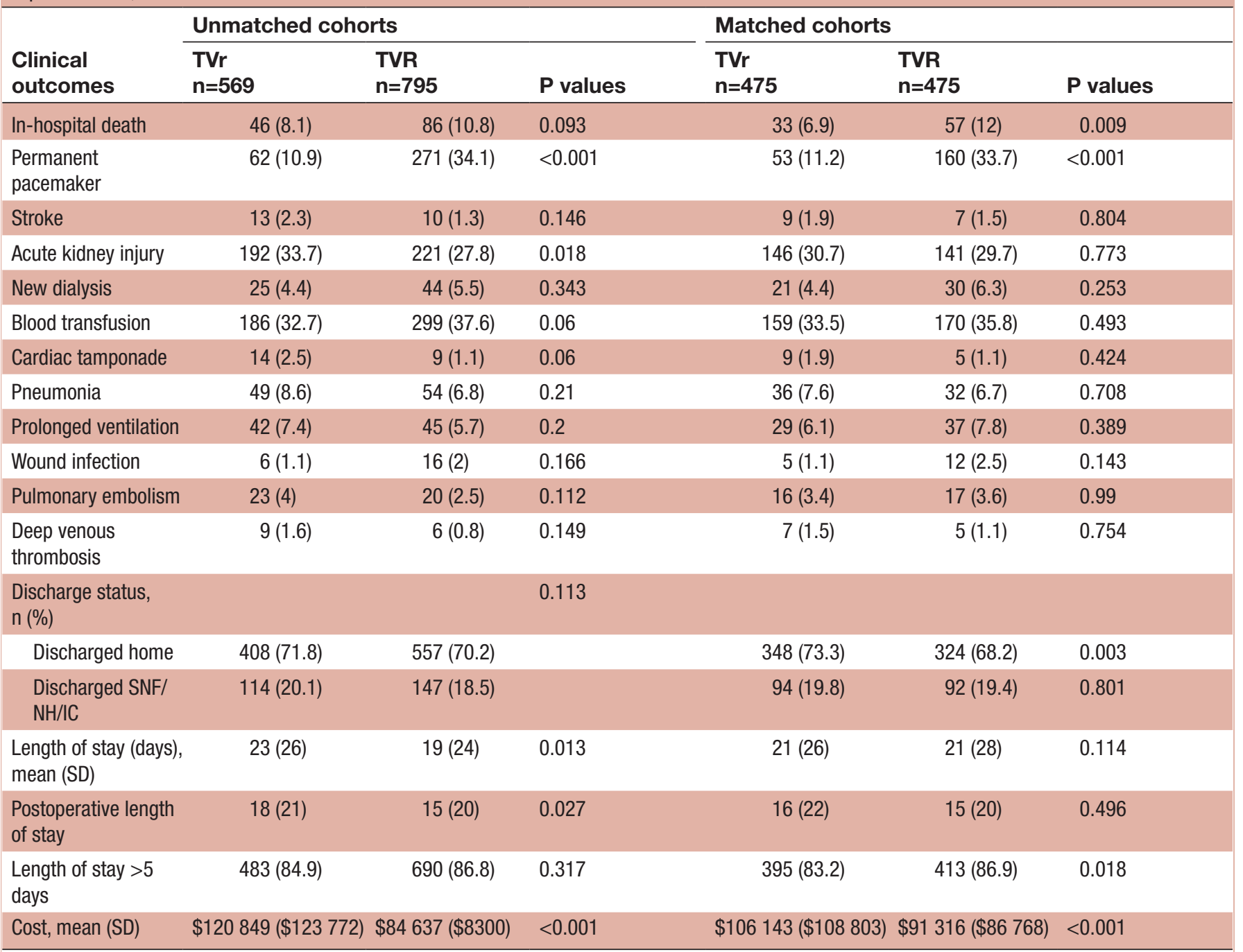

IC, intermediate care; $\mathrm{NH}$, nursing home;SNF, skilled nursing facility;TVR, tricuspid valve replacement;TVr, tricuspid valve repair. 
favourable effects of modified ultrafiltration (decreasing myocardial oedema, improving left ventricular function and improving pulmonary function compliance) have been demonstrated in non-TV cardiac surgical operations but can be quite useful in patients with TV disease due to the higher prevalence of right ventricular failure and pulmonary hypertension. ${ }^{29-31}$ Further studies are required to assess the impact of early operations, surgical techniques and various postoperative management strategies on the outcomes of TV surgery in the contemporary era.

Major postoperative complications were not significantly different between the two groups, with the exception of the rate of permanent pacemaker implantation, which was threefold higher following TVR versus TVr. Similar findings were reported in prior smaller studies. ${ }^{32} 33$ Further studies are needed to assess the potential adverse impact of permanent pacemaker implantation on long-term outcomes in these patients, especially given that one-third of patients undergoing TVR received a permanent pacemaker prior to discharge. A study by Jokinen $e t a l^{32}$ showed that patients who received permanent pacemakers following TVR experienced a higher rate of thromboembolic complications and impaired quality of life during long-term follow-up. Hospital length of stay and frequency of non-home discharges were similar between the two groups. The modestly increased cost with $\mathrm{TVr}$ was therefore unexpected, particularly with the much lower rate of pacemaker implantation in this group. We speculated the following reasons to have contributed to this cost difference: (1) The TVr group had lower in-hospital mortality and longer length of stay. The excess number of death in the TVR group might have contributed to the lesser cost as these patients would have incurred more cost should have they survived to longer hospital stays. (2) The TVr group had higher incidence of patients admitted non-electively and hence they possibly incurred additional costs associated with emergency room charges, early diagnostic testing and so on, which might have played a role in the cost difference between TVR and TVr. (3) The difference in cost, although statistically significant, is modest and may be affected by unmeasured confounders that are difficult to ascertain in administrative databases. Nonetheless, further studies are needed to elucidate the underlying aetiology of this increased cost, such as the potential influence of right ventricular failure.

\section{Limitations}

This study has several limitations. Although this is the largest nationwide study examining comparative outcomes of TVr versus TVR in patients with severe secondary TR, the results should be interpreted in the context of its limitations. (1) The NIS is a hospital claims database derived from ICD-9-CM codes and is therefore prone to coding inconsistencies. However, quality control measures are embedded in the Agency for Healthcare Research and Quality methodology to minimise these issues. In addition, the hard clinical endpoints in this study are difficult to miscode (death, pacemaker implantation, discharge status and so on). (2) We intended to study patients with secondary TR, and hence we excluded those with congenital heart disease and endocarditis. However, we cannot ascertain that these exclusion criteria were sufficient to identify a pure cohort of secondary TR. (3) A major limitation of this study is the inability to capture relevant data related to the nature and stage of TV disease (right ventricular dimension and function, pulmonary artery pressure, aetiology and duration of TV disease, left ventricular ejection fraction, and so on). We applied several layers of exclusions to arrive at a pure secondary TR population, but the lack of granular haemodynamic and echocardiographic data can possibly confound the results of the study, such as cost assessment. (4) Details on the urgency of surgery, surgical repair or replacement techniques, perioperative medications and ventilation management, and long-term outcomes beyond hospital discharge are not available in the NIS. (5) The underlying mechanism of severe TR in a non-negligible number of patients undergoing TVR is pacemaker-related TV disease. In these patients the surgery might include removal and reimplantation of permanent pacemaker leads, and hence this can potentially falsely increase the rate of permanent pacemaker implantation in the TVR group.

\section{CONCLUSIONS}

Compared with TVR, TVr is associated with at least comparable in-hospital outcomes in patients with suitable anatomy for valve repair. Further studies are needed to understand the differential impact of the type of surgery on the short-term and long-term mortality in this complex undertreated population.

Contributors MA and CB planned the study and drafted the manuscript. AK and FA conducted the analysis. VB contributed to the study's planning and made critical edits of the manuscript. MA is responsible for the overall content as the guarantor.

Funding The authors have not declared a specific grant for this research from any funding agency in the public, commercial or not-for-profit sectors.

Competing interests None declared.

Patient consent Not required.

Ethics approval The institutional review board approved the study and waived informed consent requirements because the data are derived from a nationwide deidentified database.

Provenance and peer review Not commissioned; externally peer reviewed.

Data sharing statement Data used for this analysis will be available to other researchers upon request for the purpose of reproduction of the study's results. No other unpublished data of this study are available.

Open access This is an open access article distributed in accordance with the Creative Commons Attribution Non Commercial (CC BY-NC 4.0) license, which permits others to distribute, remix, adapt, build upon this work non-commercially, and license their derivative works on different terms, provided the original work is properly cited, appropriate credit is given, any changes made indicated, and the use is non-commercial. See: http://creativecommons.org/licenses/by-nc/4.0/ 


\section{REFERENCES}

1. Tornos Mas P, Rodríguez-Palomares JF, Antunes MJ. Secondary tricuspid valve regurgitation: a forgotten entity. Heart 2015;101:1840-8.

2. Alqahtani F, Berzingi CO, Aljohani S, et al. Contemporary trends in the use and outcomes of surgical treatment of tricuspid regurgitation. J Am Heart Assoc 2017;6(12):e007597.

3. Fender EA, Zack CJ, Nishimura RA. Isolated tricuspid regurgitation: outcomes and therapeutic interventions. Heart 2018;104:798-806.

4. Choi JW, Jang MJ, Kim KH, et al. Repair versus replacement for the surgical correction of tricuspid regurgitation: a meta-analysis. Eur $J$ Cardiothorac Surg 2018;53:748-55.

5. Taramasso M, Vanermen H, Maisano F, et al. The growing clinical importance of secondary tricuspid regurgitation. J Am Coll Cardiol 2012;59:703-10.

6. Stuge O, Liddicoat J. Emerging opportunities for cardiac surgeons within structural heart disease. J Thorac Cardiovasc Surg 2006;132:1258-61.

7. Singh JP, Evans JC, Levy D, et al. Prevalence and clinical determinants of mitral, tricuspid, and aortic regurgitation (the Framingham Heart Study). Am J Cardiol 1999;83:897-902.

8. Koelling TM, Aaronson KD, Cody RJ, et al. Prognostic significance of mitral regurgitation and tricuspid regurgitation in patients with left ventricular systolic dysfunction. Am Heart J 2002;144:524-9.

9. Schueler R, Öztürk C, Sinning JM, et al. Impact of baseline tricuspid regurgitation on long-term clinical outcomes and survival after interventional edge-to-edge repair for mitral regurgitation. Clin Res Cardiol 2017;106:350-8.

10. Neuhold S, Huelsmann M, Pernicka E, et al. Impact of tricuspid regurgitation on survival in patients with chronic heart failure: unexpected findings of a long-term observational study. Eur Heart $J$ 2013;34:844-52.

11. Barbanti M, Binder RK, Dvir D, et al. Prevalence and impact of preoperative moderate/severe tricuspid regurgitation on patients undergoing transcatheter aortic valve replacement. Catheter Cardiovasc Interv 2015;85:677-84.

12. Schwartz LA, Rozenbaum Z, Ghantous E, et al. Impact of right ventricular dysfunction and tricuspid regurgitation on outcomes in patients undergoing transcatheter aortic valve replacement. J Am Soc Echocardiogr 2017;30:36-46.

13. Topilsky $Y$, Nkomo VT, Vatury $\mathrm{O}$, et al. Clinical outcome of isolated tricuspid regurgitation. JACC Cardiovasc Imaging 2014;7:1185-94.

14. Saran N, Said SM, Schaff HV, et al. Outcome of tricuspid valve surgery in the presence of permanent pacemaker. $J$ Thorac Cardiovasc Surg 2018;155:1498-508.

15. Subbotina I, Girdauskas E, Bernhardt AM, et al. Comparison of outcomes of tricuspid valve surgery in patients with reduced and normal right ventricular function. Thorac Cardiovasc Surg 2017;65:617-25.

16. Redondo Palacios A, López Menéndez J, Miguelena Hycka J, et al. Which type of valve should we use in tricuspid position? Long-term comparison between mechanical and biological valves. J Cardiovasc Surg 2017;58:739-46.
17. Lange R, Piazza N, Günther T. [Tricuspid valve regurgitation: indications and operative techniques]. Herz 2017;42:653-61.

18. Singh SK, Tang GH, Maganti MD, et al. Midterm outcomes of tricuspid valve repair versus replacement for organic tricuspid disease. Ann Thorac Surg 2006;82:1735-41. discussion 41.

19. Marquis-Gravel G, Bouchard D, Perrault LP, et al. Retrospective cohort analysis of 926 tricuspid valve surgeries: clinical and hemodynamic outcomes with propensity score analysis. Am Heart $J$ 2012;163:851-8.

20. Moraca RJ, Moon MR, Lawton JS, et al. Outcomes of tricuspid valve repair and replacement: a propensity analysis. Ann Thorac Surg 2009;87:83-9. discussion 8-9..

21. Hwang HY, Kim KH, Kim KB, et al. Treatment for severe functional tricuspid regurgitation: annuloplasty versus valve replacement. Eur J Cardiothorac Surg 2014;46:e21-7.

22. Chang HW, Jeong DS, Cho $\mathrm{YH}$, et al. Tricuspid valve replacement vs. repair in severe tricuspid regurgitation. Circ J 2017;81:330-8.

23. McGrath LB, Gonzalez-Lavin L, Bailey BM, et al. Tricuspid valve operations in 530 patients. Twenty-five-year assessment of early and late phase events. J Thorac Cardiovasc Surg 1990;99:124-33.

24. Guenther T, Noebauer C, Mazzitelli D, et al. Tricuspid valve surgery: a thirty-year assessment of early and late outcome. Eur J Cardiothorac Surg 2008;34:402-9. discussion 9.

25. Kim JB, Jung SH, Choo SJ, et al. Surgical outcomes of severe tricuspid regurgitation: predictors of adverse clinical outcomes. Heart 2013;99:181-7.

26. Raikhelkar J, Lin HM, Neckman D, et al. Isolated tricuspid valve surgery: predictors of adverse outcome and survival. Heart Lung Circ 2013;22:211-20.

27. De Meester P, Van De Bruaene A, Voigt JU, et al. Outcome and determinants of prognosis in patients undergoing isolated tricuspid valve surgery: retrospective single center analysis. Int J Cardiol 2014;175:333-9.

28. Jeong DS, Park PW, Mwambu TP, et al. Tricuspid reoperation after left-sided rheumatic valve operations. Ann Thorac Surg 2013;95:2007-13.

29. Sung K, Park PW, Park KH, et al. Is tricuspid valve replacement a catastrophic operation? Eur J Cardiothorac Surg 2009;36:825-9.

30. Luciani GB, Menon T, Vecchi B, et al. Modified ultrafiltration reduces morbidity after adult cardiac operations: a prospective, randomized clinical trial. Circulation 2001;104(12 Suppl 1):I253-9.

31. Yie K, Yang HS. Early operative mortality with tricuspid valve replacement does not simply depend on surgery itself; late-tricuspid regurgitation is a marker of late-stage myocardial and valvular heart disease. European Journal of Cardio-Thoracic Surgery 2010;37:1477. author reply 8.

32. Jokinen JJ, Turpeinen AK, Pitkänen $\mathrm{O}$, et al. Pacemaker therapy after tricuspid valve operations: implications on mortality, morbidity, and quality of life. Ann Thorac Surg 2009;87:1806-14.

33. Do QB, Pellerin M, Carrier M, et al. Clinical outcome after isolated tricuspid valve replacement: 20 -year experience. Can J Cardiol 2000;16:489-93. 